For the definitive version of this article, please refer to:

Chey, M.H., Chase, J.G., Mander, J.B. and Carr, A.J. (2010) Semi-active tuned mass damper building systems: Application. Earthquake Engineering \& Structural Dynamics (EESD), 39(1), 69-

89. http://dx.doi.org/10.1002/eqe.933

\title{
Semi-active tuned mass damper building systems: Application
}

\author{
Min-Ho Chey ${ }^{1}$, J. Geoffrey Chase ${ }^{2 * \dagger \neq}$, John B. Mander ${ }^{3}$, Athol J. Carr ${ }^{1}$ \\ ${ }^{1}$ Department of Civil and Natural Resources Engineering, University of Canterbury, Private Bag 4800, Christchurch, New Zealand \\ ${ }^{2}$ Department of Mechanical Engineering, University of Canterbury, Private Bag 4800, Christchurch, New Zealand \\ ${ }^{3}$ Zachry Department of Civil Engineering, Texas A\&M University, TAMU 3136, College Station, TX 77843-3136, USA
}

\begin{abstract}
SUMMARY
Seismic performance attributes of multi-story passive and semi-active tuned mass damper (PTMD and SATMD) building systems are investigated for 12-story moment resisting frames modeled as ' $10+2$ ' stories and ' $8+4$ ' stories. Segmented upper portion of the stories are isolated as a tuned mass, and a passive viscous damper or semi-active resetable device is adopted as energy dissipation strategy. The semi-active approach uses feedback control to alter or manipulate the reaction forces, effectively re-tuning the system depending on the structural response. Optimum TMD control parameters and appropriate matching SATMD configurations are adopted from a companion study on a simplified two-degree-of-freedom (2-DOF) system. Statistical performance metrics are presented for 30 probabilistically scaled earthquake records from the SAC project. Time history analyses are used to compute response reduction factors across a wide range of seismic hazard intensities. Results show that large SATMD systems can effectively manage seismic response for multi-degree-of freedom (MDOF) systems across a broad range of ground motions in comparison to passive solutions. Specific results include the identification of differences in the mechanisms by which SATMD and PTMD systems remove energy, based on the differences in the devices used. Additionally, variability is seen to be tighter for the SATMD systems across the suites of ground motions used, indicating a more robust control system. While the overall efficacy of the concept is shown the major issues, such as isolation layer displacement, are discussed in details not available in simplified spectral analyses, providing further insight into the dynamics of these issues for these systems.
\end{abstract}

KEY WORDS: tuned mass damper; segmental building; semi-active control; resetable device; seismic hazard; statistical assessment

\footnotetext{
*Correspondence to: J. Geoffrey Chase, Department of Mechanical Engineering, University of Canterbury, Private Bag 4800, Christchurch, New Zealand.

† E-mail: geoff.chase@canterbury.ac.nz

${ }^{\ddagger}$ Professor
} 


\section{INTRODUCTION}

Tuned mass damper (TMD) systems are often considered as a practical seismic response control solution for flexible structures, such as tall buildings [1-3]. The main disadvantage of a TMD system is the sensitivity related to the narrow band control and the fluctuation in tuning the TMD frequency to the controlled frequency of a potentially degrading structure. Another limitation of a TMD is the size of the tuned absorber mass. In an attempt to increase the performance of the TMD without incurring the problems noted above, active and semi-active TMD (ATMD and SATMD) systems have been proposed [4-7]. To overcome the limitation of the TMD mass, various seismic isolation concepts using TMD related principles have been extended to convert a structural system into a TMD system by specially designing the structural system [8-16].

As a separate part of the present research, simplified PTMD and SATMD building models were presented and implemented in a system design simulation [17]. In the trade-off parametric study of this approach, the efficiency of spreading stiffness between resetable devices and rubber bearings was illustrated. The end result of this spectral analysis is an optimally-based initial design approach that fits into accepted design methods, and the efficiency of these modified control system and the validity of the optimal designs were demonstrated as the reference for MDOF verification. In the view point of traditional TMD system theory, this alternative approach avoids adding excessive redundant mass that is rarely used.

This paper examines multi-story SATMD building systems that use segregated upper stories as relatively very large tuned masses and semi-active devices to provide robust adaptability to broader ranges of structural response. For this study, the performance of 12-story SATMD building system models are compared with those from the corresponding uncontrolled (No TMD) and PTMD building systems subjected to the suites of probabilistically scaled ground motions. Results are presented using appropriate log-normal statistics so that results can be considered as part of a standard hazard and design framework. The goal of this research is to validate the MDOF analyses, the overall robustness and efficiency of this SATMD design concept in comparison to equivalent, widely accepted passive TMD (PTMD) system.

\section{12-STORY TMD BUILDING SYSTEMS}

\subsection{Structural modeling}

To demonstrate the effects of the TMD (PTMD and SATMD) building system, realistic 12-story two-bay reinforced concrete framed structure models have been developed in Ruaumoko [18]. This model was designed originally by Jury [19] according to the New Zealand Loadings Code [20] based on the concept of capacity design. Its strengths were then revised following the changes to the Code made in 1992 [21].

According to the NZS Code for beam design, all frames share in carrying gravity and seismic-induced loads. Moment redistribution is then carried out using a method developed by Paulay [22]. An effort was made during moment redistribution to allow the full utilization of beam sections by equalizing, if possible, the demand for top and bottom flexural steel at the column face. Thomson [23] increased the dimensions 
according to data reported by Paulay [24], because the original design [19] called for unrealistically high reinforcement ratios. The columns above the first level were specified to remain elastic in accordance with the strong column-weak beam concept.

It was assumed that the frame would be required to resist the component of earthquake motion in the plane of the frame only. No torsional effects for the building as a whole were taken into account. The columns above the first level were specified to remain elastic in accordance with the strong column - weak beam concept. A width of the floor slab equal to 12 times its thickness was considered to contribute to the elastic stiffness of the beams. The slab thicknesses were $120 \mathrm{~mm}$ for the framed structure.

For the PTMD and SATMD systems, the upper two and four stories are isolated respectively. The resulting retrofitted structures are modeled as ' $10+2$ ' story and ' $8+4$ ' story structures, as shown in Figure 1(a). Figure 1(b) shows the schematic of the isolation layer including rubber bearings and viscous damper (PTMD) or resetable device (SATMD).

The natural period of the lower part of the each frame model is $1.52 \mathrm{sec}$ for the 10 story structure and 1.19sec for the 8-story structure respectively. The structural damping ratio of each structure is assumed to be $5 \%$ of critical damping. The total weight of the TMD building structures (10+2 and $8+4$ structures) is 19.2MN (mass $~ 2,000$ tones). The dynamic properties of the frames, including modal characteristics are listed in Table 1, and the building dimensions and member sizes adopted in these models are shown in Table 2.

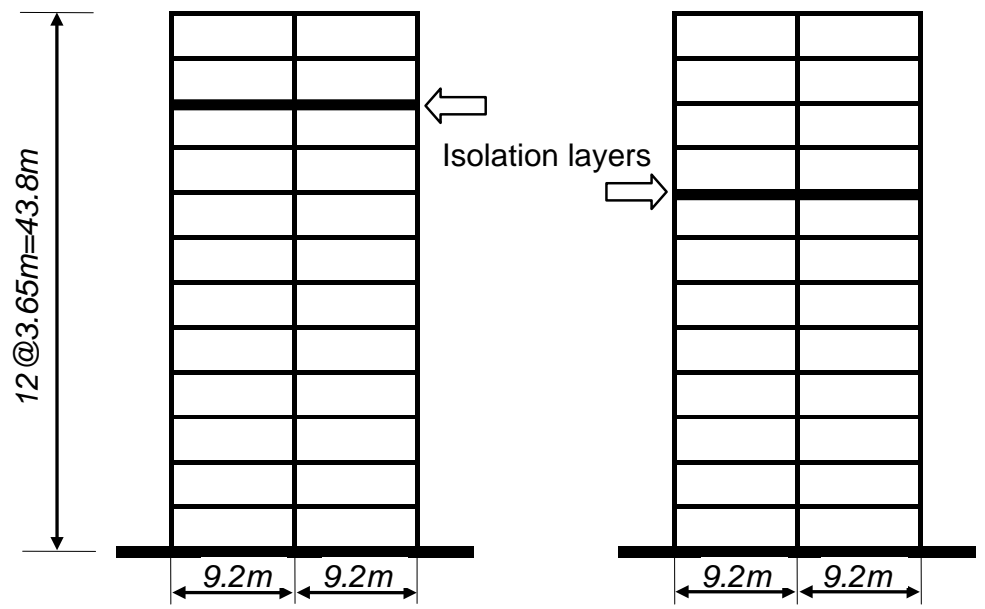

(a) ' $10+2$ ' and ' $8+4$ ' models of 12-story two-bay reinforced concrete frames

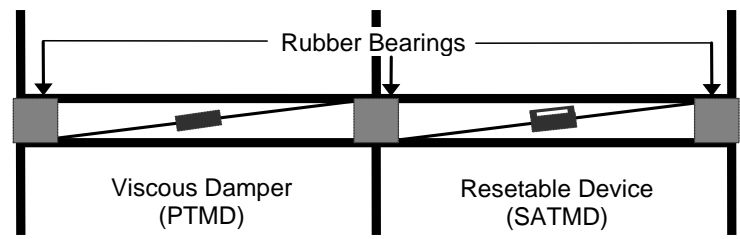

(b) Schematic of isolation layer

Figure. 1. The segmented 12-story buildings studied 


\subsection{Parametric control of TMD}

For the optimum TMD parameters, it was found that the tuning ratio for a MDOF system is nearly equal to the tuning ratio for a 2-DOF system for a mass ratio of $\mu \Phi$, where $\Phi$ is the amplitude of the first mode of vibration for a unit modal participation factor computed at the location of the TMD [2]. The equation for the tuning ratio is thus obtained from the equation for the 2-DOF system by replacing $\mu$ by $\mu \Phi$.

$$
f_{M 2 o p t}=\frac{1}{1+\mu \Phi}\left(1-\xi_{1} \sqrt{\frac{\mu \Phi}{1+\mu \Phi}}\right)
$$

The TMD damping ratio is also found to correspond approximately to the damping ratio computed for a 2-DOF system multiplied by $\Phi$. Thus, the equation for the damping ratio is obtained by multiplying the equation for the 2-DOF system by $\Phi$.

$$
\xi_{M 2 o p t}=\Phi\left(\frac{\xi_{1}}{1+\mu}+\sqrt{\frac{\mu}{1+\mu}}\right)
$$

For the MDOF structures, the practical parameters of the optimal TMD stiffness and the optimal damping coefficient can therefore be derived respectively as

$$
\begin{gathered}
k_{M 2 o p t}=m_{2} \omega_{1}^{2} f_{M 2 o p t}{ }^{2}=\frac{m_{2} \omega_{1}^{2}}{(1+\mu \Phi)^{2}}\left(1-\xi_{1} \sqrt{\frac{\mu \Phi}{1+\mu \Phi}}\right)^{2} \\
c_{M 2 o p t}=2 m_{2} \omega_{1} f_{M 2 o p t} \xi_{M 2 o p t}=\frac{2 m_{2} \omega_{1}}{1+\mu \Phi}\left(1-\xi_{1} \sqrt{\frac{\mu \Phi}{1+\mu \Phi}}\right)\left(\frac{\xi_{1}}{1+\mu}+\sqrt{\frac{\mu}{1+\mu}}\right)
\end{gathered}
$$

Figure 2(a) shows the optimum passive TMD tuning and damping ratios against mass ratios of 0 to 1 with $5 \%$ of internal damping for $10+2$ and $8+4$ story models. The optimum values for the $10+2$ and $8+2$ models examined here have been marked by small squares on the lines at the mass ratios of 0.244 and 0.594 respectively. For the $10+2$ and $8+4$ models, the masses of the primary structures are 1,640t (10-story) and 1,320t (8-story), and the amplitude of the first modal vibration, $\Phi$, of 1.343 and 1.309 are adopted, respectively. Figure 2(b) shows the optimum passive TMD stiffness and damping coefficient for the models of $10+2$ and $8+4$ cases. It can be seen that the gaps between the optimum TMD stiffness lines for the two models are increased with the increase of mass ratio. However, just small gaps can be found between the optimum TMD damping coefficients for the two models. 


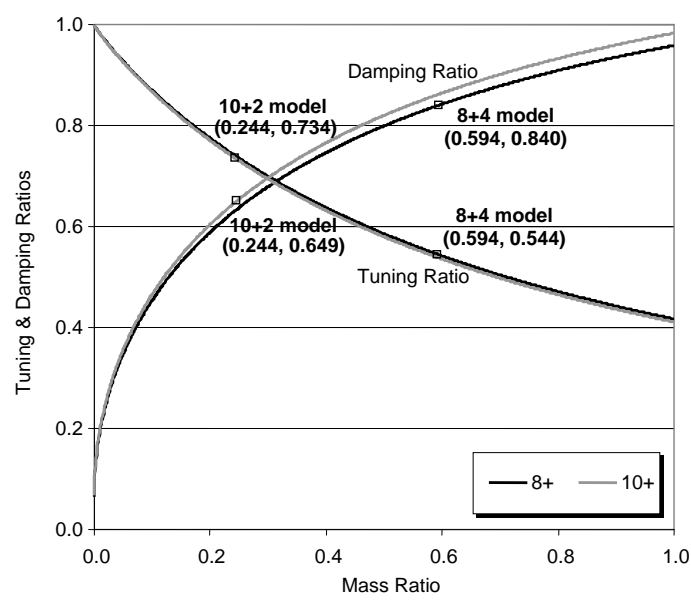

(a) TMD tuning and damping ratios

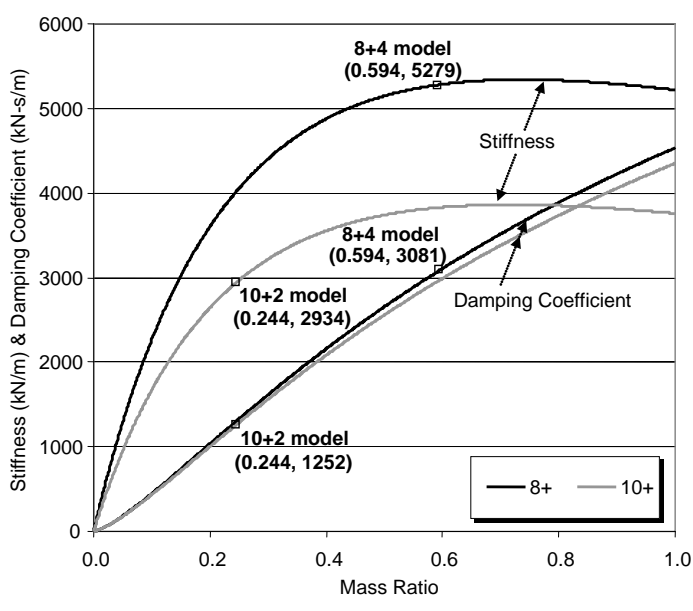

(b) TMD stiffness and damping coefficient

Figure 2. Optimum TMD parameters for different mass ratios (10+2 and 8+4 models with 5\% of critical damping)

It was noted that under the considered structural properties and the ground excitations, the linear displacement response due to the first mode constitutes approximately $80 \% \sim 90 \%$ of the total displacement response. Thus, the first mode is selected for the designs of the PTMD and SATMD systems. The resulting optimum parameters are listed in Table 3 . The total value of $k_{M 2 o p t}$ is allocated to rubber bearing stiffness and the stiffness of the SA resetable device. The SATMD, having the same stiffness values of the resetable device and rubber bearings, has been chosen and adopted for each structure and earthquake suite (SATMD $=k_{\text {M2opt }}$ with an added small coupling stiffness less than 5\%). This equivalent combined stiffness was chosen for simplicity and may not represent an optimal SATMD design [25]. However, this stiffness value might represents a good compromise in reducing the response value and bandwidth across the three earthquake suites.

From the parametric results, the design range of effective SATMD system is derived and then adopted as a practical control scheme. Note that a more optimal SATMD parameter combinations may be available. In fact, their control ability is improved when a stiffness lower than the optimal value is used, providing a more robust and effective seismic energy management. The SATMD system provides a better, more robust overall control strategy than PTMD system, especially if the optimum stiffness of TMD is not ideal or perfectly tuned. Semi-active solutions are also not constrained by the optimum tuning stiffness for the TMD like the passive case. Thus, the SATMD system is easier to design as no tuning is required. In contrast, slight "out-of-tuning" in the passive PTMD case can have a significant detrimental effect on the controlled response.

For convenience, a flow diagram of optimal design of MDOF TMD building system by numerical optimization is shown in Figure 3. From the diagram, it is seen that the TMD parameters are based on the results of the 2-DOF design process. The modified TMD parameters for the MDOF system are applied to the multi-story structures. The dynamic characteristics of the controlled systems are analyzed by modal analysis. Finally, time history analyses using suites of ground motions supplies the individual performance values for the final statistical performance assessment, since the use of a probabilistic format allows for a consideration of structural response over a range of seismic hazards. 


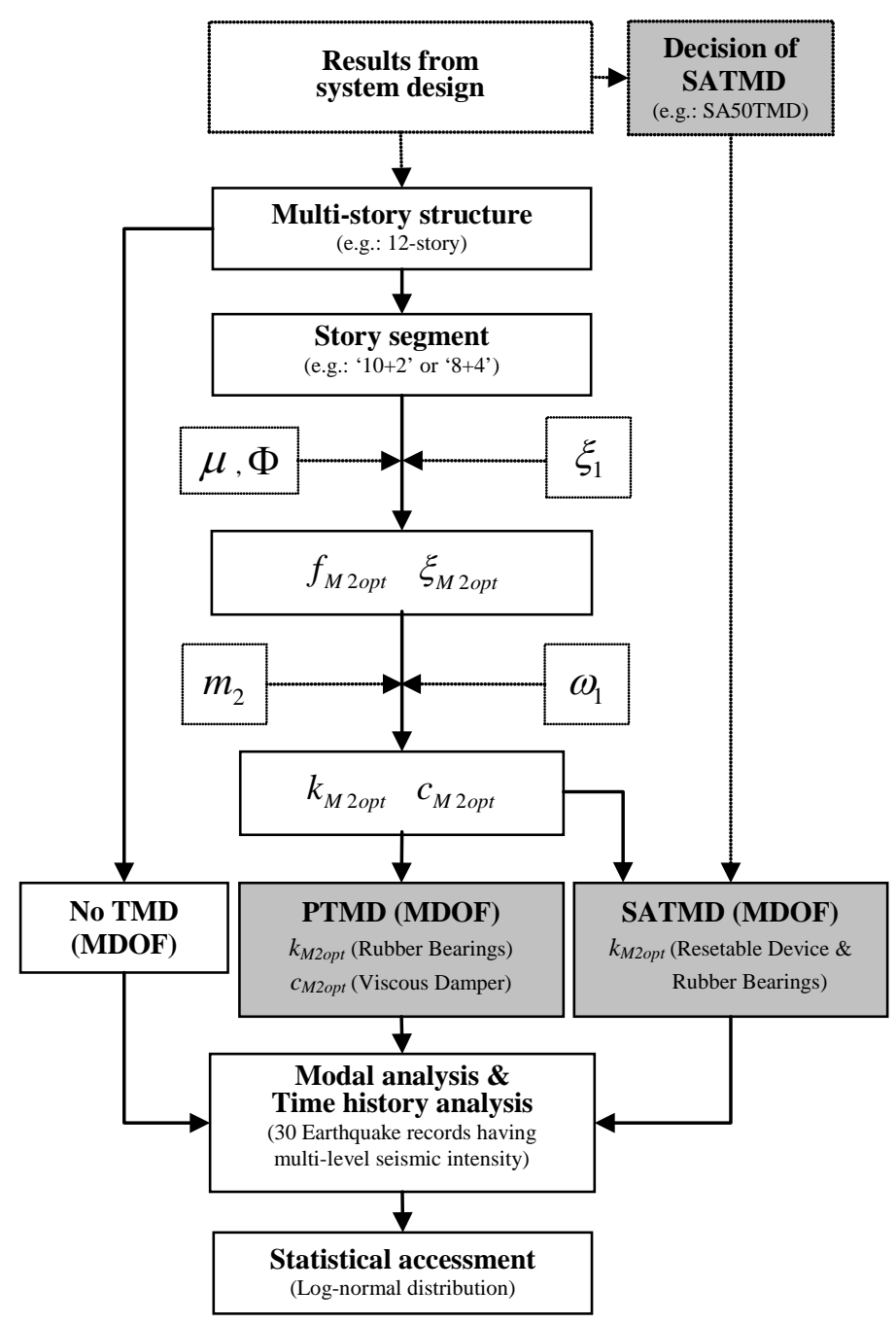

Figure 3. Verification process for the TMD building system

\subsection{Dynamic characteristics of resetable device}

For a given structural configuration, the connections between the segregated (upper) structure and main (lower) structure can be semi-active resetable device for a 12-story SATMD building system, and the design of resetable device is based on the previous results of the system design [17]. For these cases, the maximum device forces are set at $644 \mathrm{kN}$ and $1,573 \mathrm{kN}$, which represent the value of $13.8 \%$ [26] of the structural weight multiplied by mass ratios of $0.244(10+2)$ and $0.594(8+4)$, respectively.

Figure 4 shows the force-displacement loops for a modeled, ideal SATMD under three different levels of earthquake intensity. This form is denoted a '1-4 device' as it provides damping in all quadrants $[27,28]$. All stored energy is released at the peak of each sine-wave cycle and all other motion is resisted [29]. The force-displacement loops show that the force grows linearly with displacement until the maximum displacement is reached. At this point, the force drops indicating that the device has reset. The force then decreases linearly with decreasing displacement until the minimum is reached at which the force jumps to zero again showing that the device has once again reset. These 
loops represent basic, idealized resetable device operations [26, 29-31].

New results in resetable devices can provide highly customized hysteresis loops [27, 28]. It has introduced a novel device design with independent chambers that disassociates the chamber pressures resulting in control law applications not possible for the original [31] proposed design. For this standard case, devices with up to $1.7 \mathrm{MN}$ are already in use in limited numbers for commercial structures [32, 33]. Hence, the devices for this approach may be assumed to be either available or within the possibility to design.
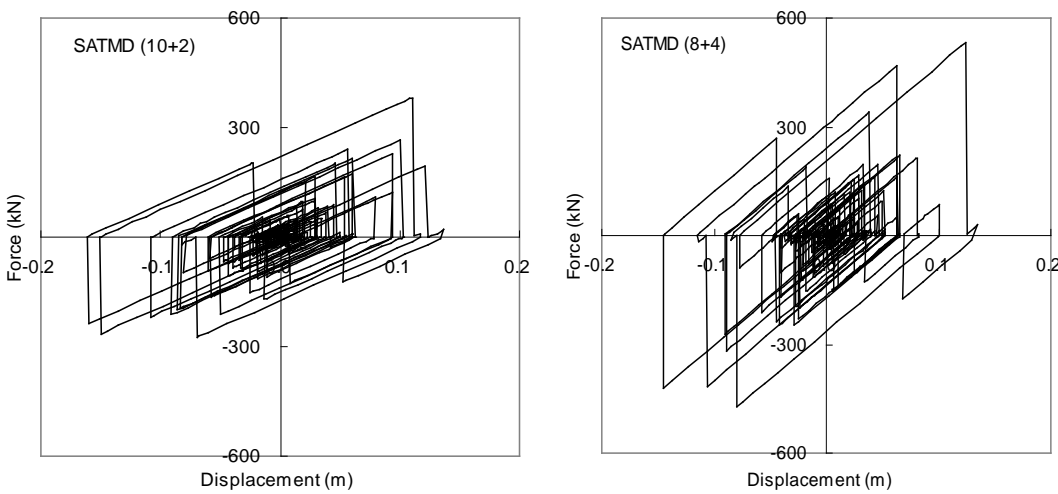

(a) Kern County (Low Suite)
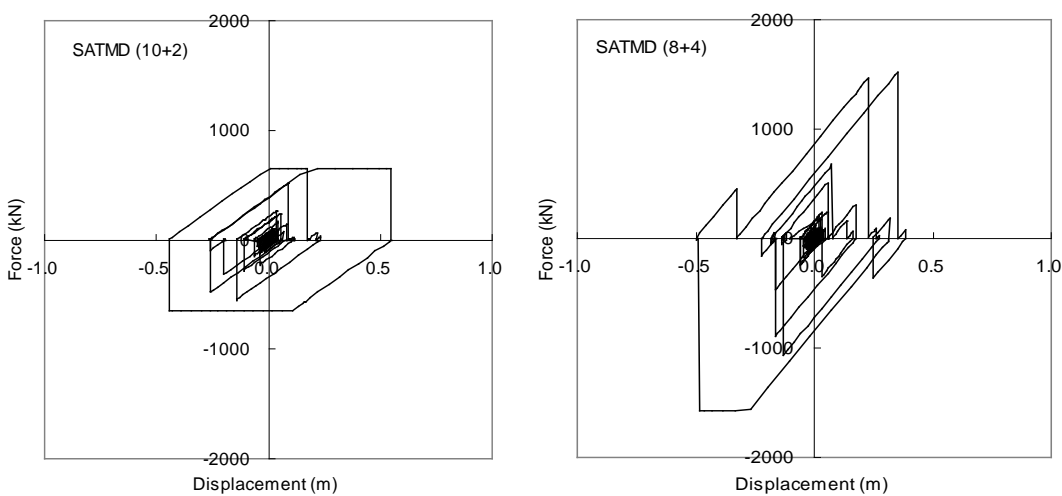

(b) Imperial Valley (Medium Suite)
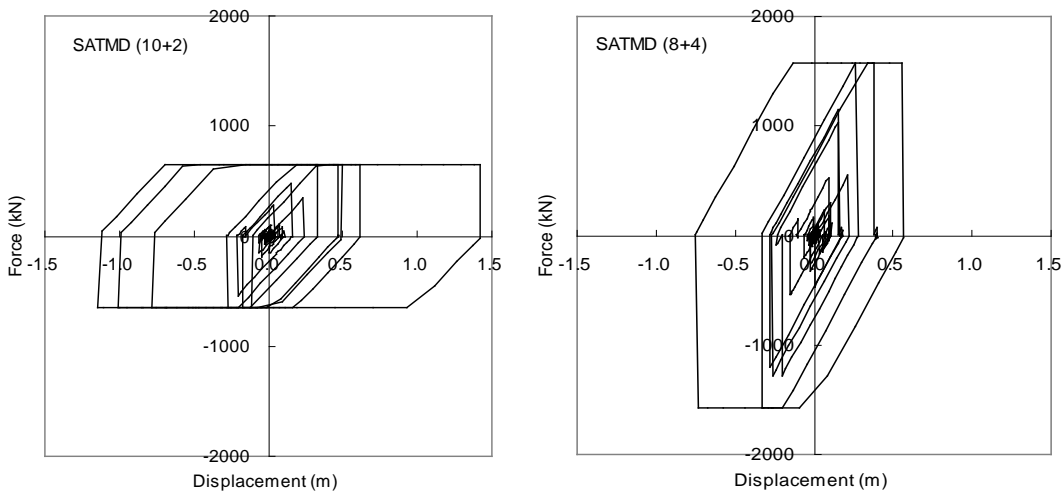

(c) Kobe (High Suite)

Figure 4. Hysteretic behavior of resetable device 


\section{STRUCTURAL ANALYSIS}

\subsection{Modal analysis}

Modal analysis results using Ruaumoko are shown in Figure 5. The TMD building systems here offer two main modes of vibration, while just one in the 12-story uncontrolled (No TMD) case. Despite having two main modes and thus a system susceptible to receiving larger amounts of input energy from the earthquake, a relatively large portion of the entrapped energy is concentrated on the isolation layer [15]. For the SATMD building systems, the first mode dominates the upper stories and a much smaller magnitude second mode dominates lower stories. Thus the both first and second modes are decoupled by the isolation layer.
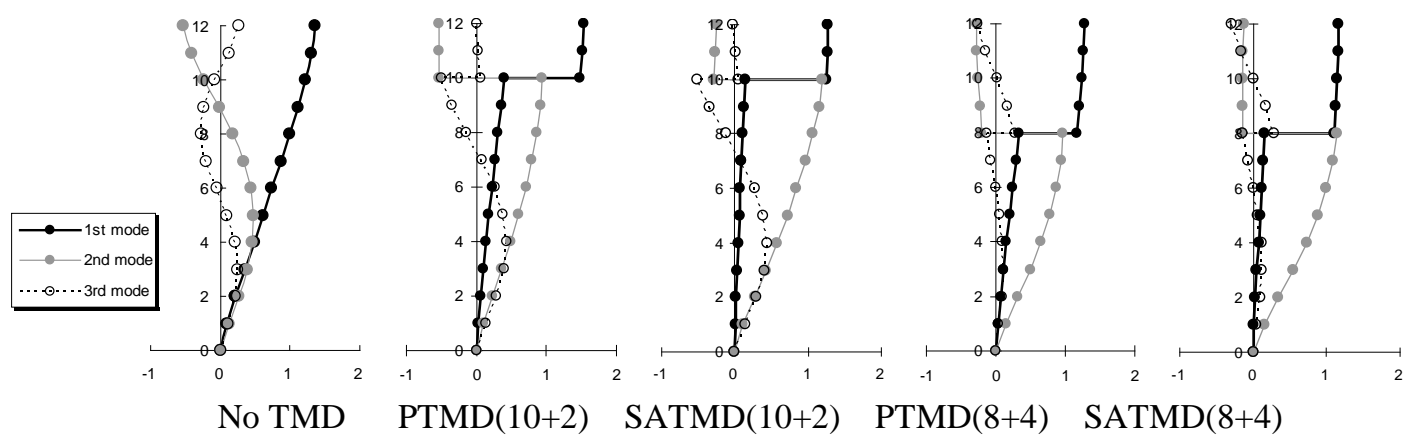

Figure 5. Modal analysis of the TMD building systems

These results indicate two different methods of dissipating energy. The PTMD dissipates energy via tuned absorption, while the SATMD dissipates energy via enhanced relative motion obtained by decoupling the segments.

The parameter of modal participation factor for $i^{\text {th }}$ mode is defined as

$$
\Gamma_{i}=\frac{L_{i}}{M_{i}}
$$

in which $L_{i}$ is the earthquake excitation factor for $i^{\text {th }}$ mode, and $M_{i}$ is the generated modal mass of that mode. Another useful parameter for the modal analysis is the mass participation factor.

$$
\beta_{i}=\frac{M_{e f f, i}}{M}=\frac{1}{M} \frac{L_{i}^{2}}{M_{i}}
$$

in which $M_{\text {eff }, i}$ is the effective mass for the $i^{\text {th }}$ mode and $M$ is the total mass of the building. Because the effective mass indicates the importance of the contribution of the $i^{\text {th }}$ mode to the total base shear acting on the structure, the mass participation factor can be an index showing how much of the total mass of the building will contribute in generating base shear in that mode [9]. So, if the mass participation factor of the first 
mode is much higher than that of the second mode, the first mode can be readily excited by base excitation.

Table 4 shows the numerical results of the modal analysis. Second modal participation factors of the SATMD $(10+2$ and $8+4)$ building systems are closer to those of first mode and relatively larger than those of the second mode of PTMD. Furthermore, the second mass participation factors of the SATMD building systems are larger than those of the first mode. Therefore, in the SATMD building system, the interaction between the first and second modes is more pronounced and the relatively larger mode and mass participations of the second mode for the SATMD building system may contribute to the further reduction of the overall responses of displacement and base shear responses compared to the PTMD results.

It should be noted that the factor $\Phi$ used in Section 2.2 to define an equivalent MDOF optimal TMD stiffness and damping may be less applicable [2] when larger mass ratios are used, as is the case here. Certainly, given Figure 5, the mode shapes are significantly disturbed from the original No TMD case. However, the validity of the analysis from [2] should hold for two main reasons. First, the mode shapes in Figure 5 are similar up to the break in structural sections, above which the effectively rigid TMD section behaves differently, thus the relative motions up to that point will hold. Second, the tuning used in Section 2.2 is based on modal participation, rather than mode shape specifically, where the $1^{\text {st }}$ and $2^{\text {nd }}$ modes still dominate response in this case as well. However, a further, more detailed analysis of this point would be needed to clarify this issue explicitly to ensure no loss of PTMD optimality in the comparison. That said, it should be noted that the SATMD systems are compared to the PTMD systems based on this TMD stiffness so the comparison across systems is equally handicapped. Thus, if this aspect is not optimal, this limitation of the analysis should be mitigated based on the comparison used.

Finally, note that the modal analysis is done including the device stiffness, or device is seen as being on. Hence, Figure 5 shows that, normalization aside, the mode shapes for the system are effectively the same as the No TMD case up to the break between base and TMD portions for PTMD and SATMD. Then the SATMD and PTMD systems have a discontinuity at the isolation layer (see Figure 1) where similar stiffnesses are used. Thus, the PTMD and SATMD systems have highly similar mode shapes. The remaining differences are attributed to the specifics of how the finite element code manages each element type, where the resetable device elements (see Figure 4) are axial elements in their connection between stories transmitting only that axial behavior to the 3 local translations at each story, and the springs for the PTMD are connected directly between all 3 translations.

\subsection{Time history analysis}

The structural analysis is performed using the three (low, medium and high) suites of ground motions from the SAC project [34]. To indicate the spread of results the $50^{\text {th }}$ (median) and $84^{\text {th }}$ (median +1 lognormal standard deviation) percentiles are presented using lognormal statistics [26, 35]. Figures 6-8 show the results of seismic response subjected to the three suites of earthquakes for the No TMD (12-story), PTMD (10+2 and $8+4)$ and SATMD $(10+2$ and $8+4)$ structures. For comparison, the SATMD $(8+4)^{*}$ which used $33 \%$ of optimum TMD stiffness (without rubber bearing) is also presented. 
The maximum relative displacements, interstory drift ratios, normalized story shear forces (shear forces divided by structure weight) and total accelerations for all floors are calculated as the evaluation criteria of the control effectiveness.

To compare the relative ability of the different TMD building systems at reducing the seismic demands, the $50^{\text {th }}$ percentile (median) response reduction factor profiles for each suite are presented for the PTMD $(10+2$ and $8+4)$ and SATMD $(10+2$ and $8+4)$ building systems in series of results presented in Figures 9 to 11. The multiplicative reduction factors shown in these figures are normalized to the corresponding uncontrolled (No TMD) floor response values. For the response performance indices presented, the reduction factor profiles clearly indicate the advantage of the structural operation of the PTMD and SATMD building systems. 

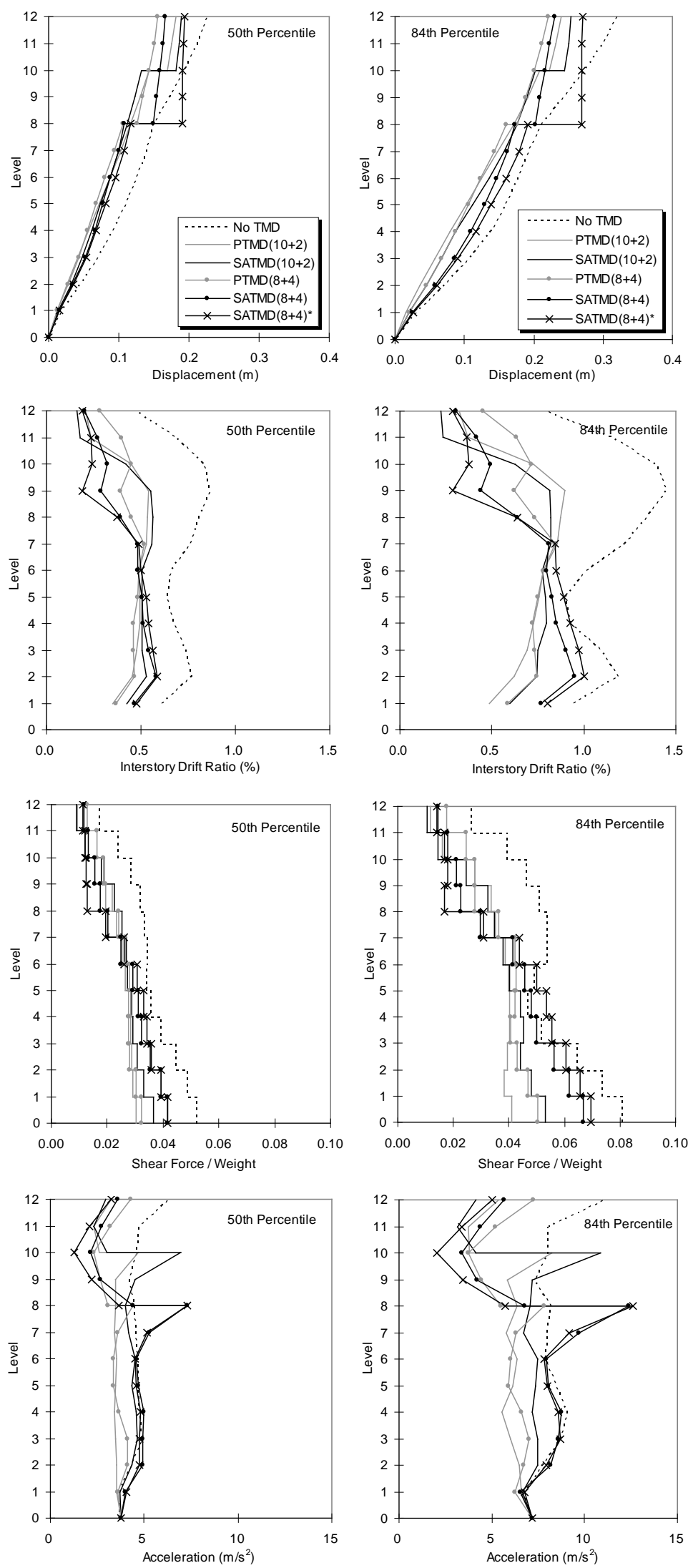

Figure 6. Low suite performance of ' $10+2$ ' and ' $8+4$ ' models ( $50^{\text {th }}$ (median) and $84^{\text {th }}$ percentiles) 

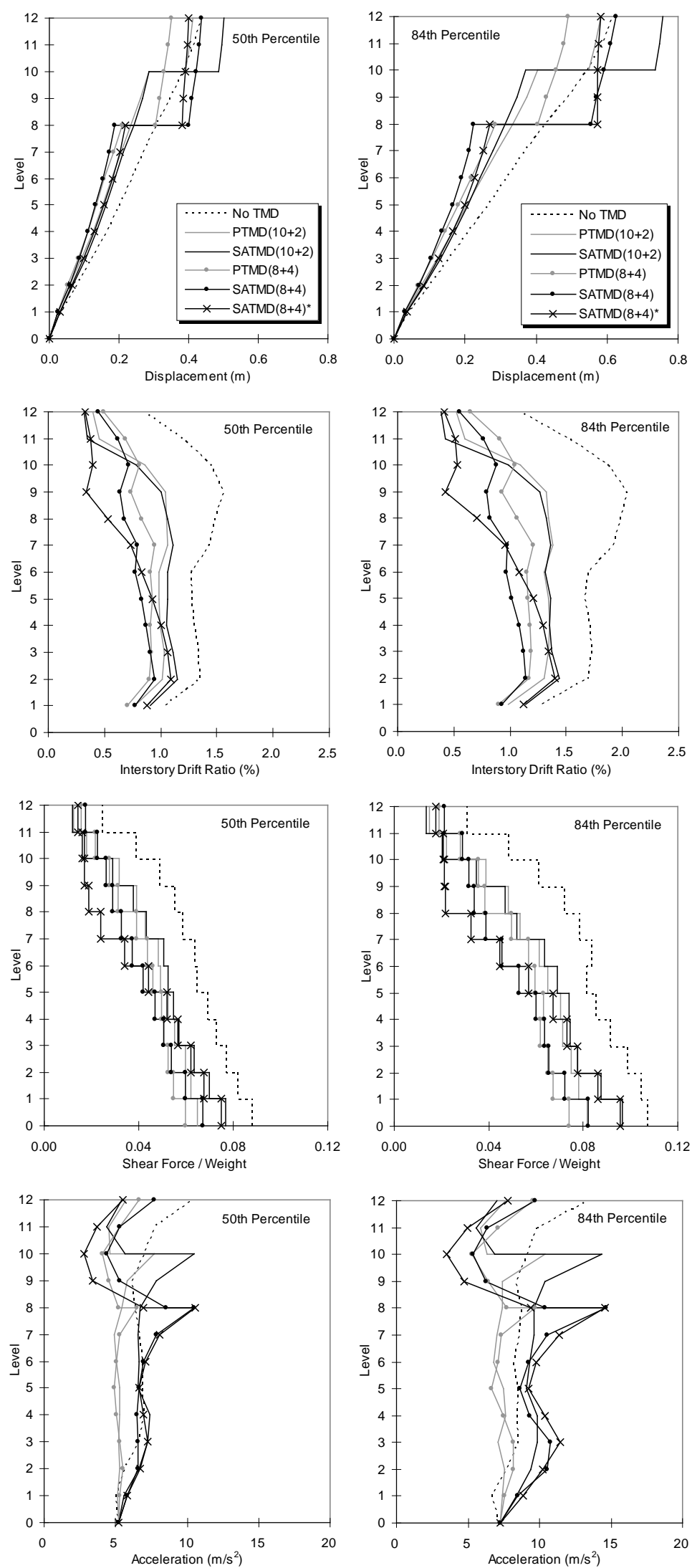

Figure 7. Medium suite performance of ' $10+2$ ' and ' $8+4$ ' models ( $50^{\text {th }}$ (median) and $84^{\text {th }}$ percentiles) 

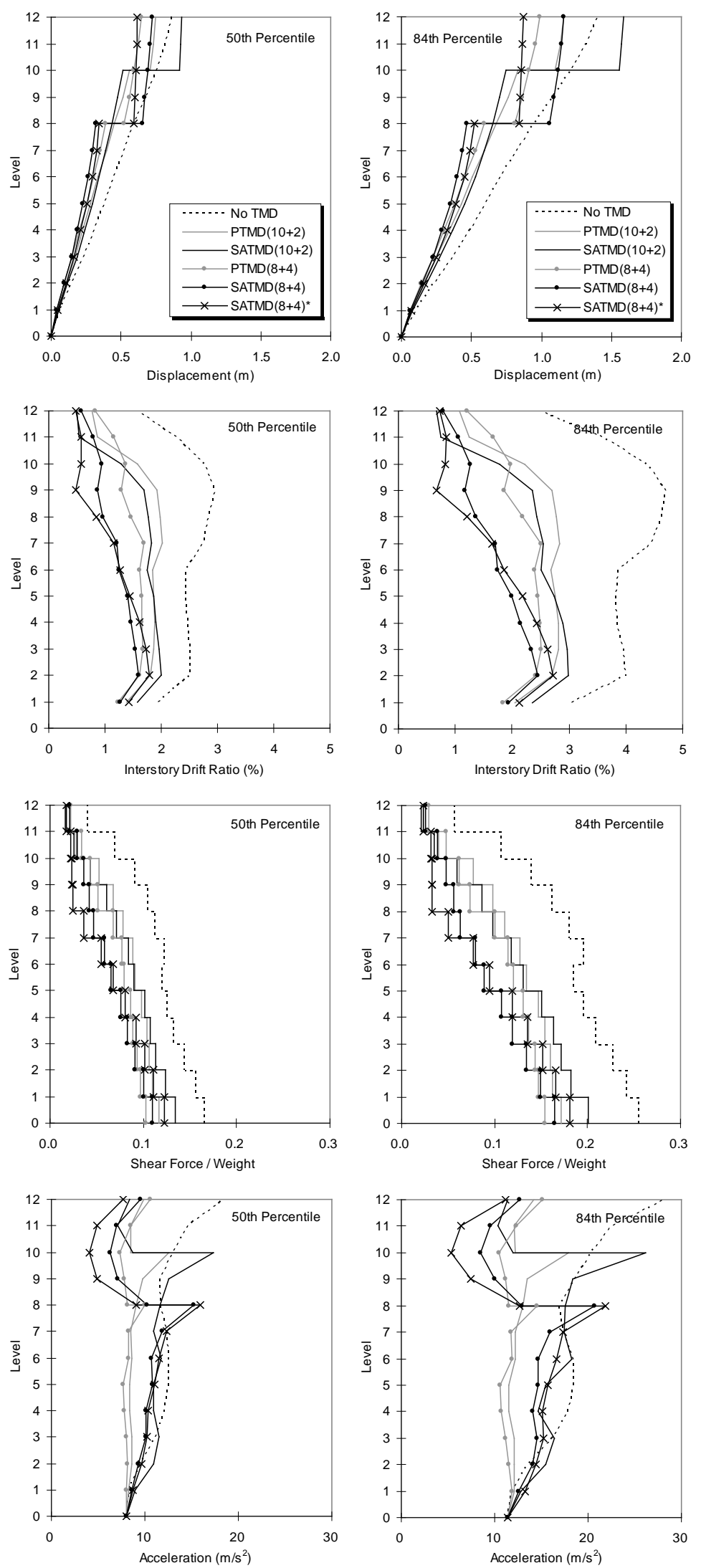

Figure 8. High suite performance of ' $10+2$ ' and ' $8+4$ ' models $\left(50^{\text {th }}\right.$ (median) and $84^{\text {th }}$ percentiles) 

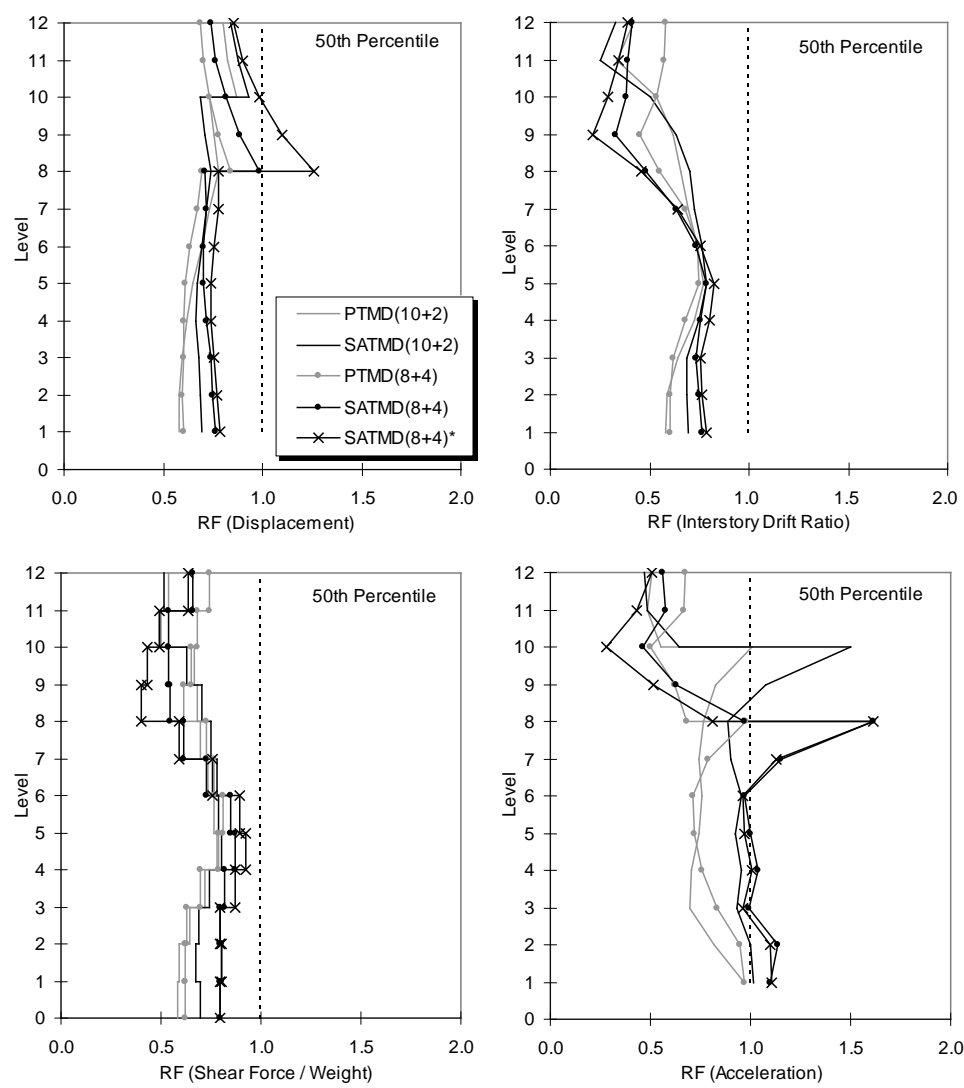

Figure 9. Low suite response reduction factors of ' $10+2$ ' and ' $8+4$ ' models ( $50^{\text {th }}$ percentile)
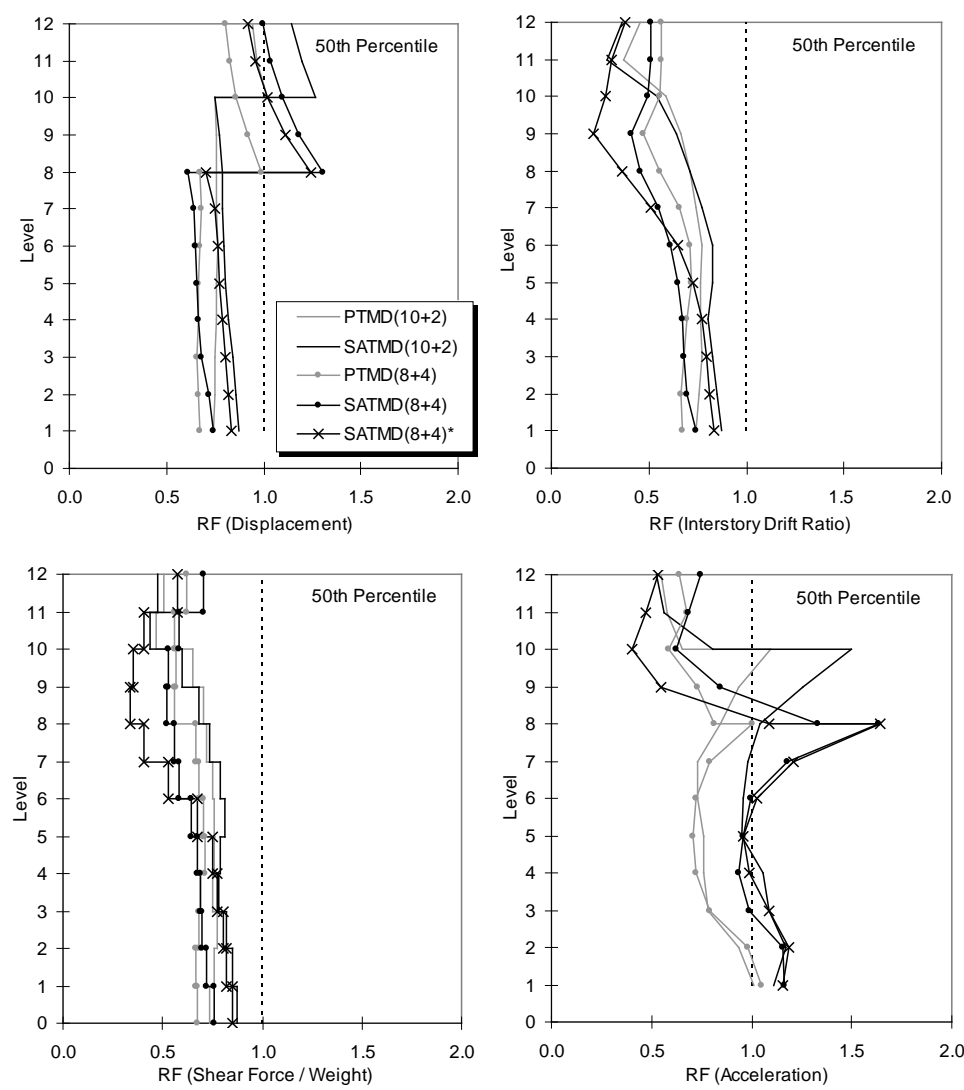

Figure 10. Medium suite response reduction factors of ' $10+2$ ' and ' $8+4$ ' models $\left(50^{\text {th }}\right.$ percentile) 

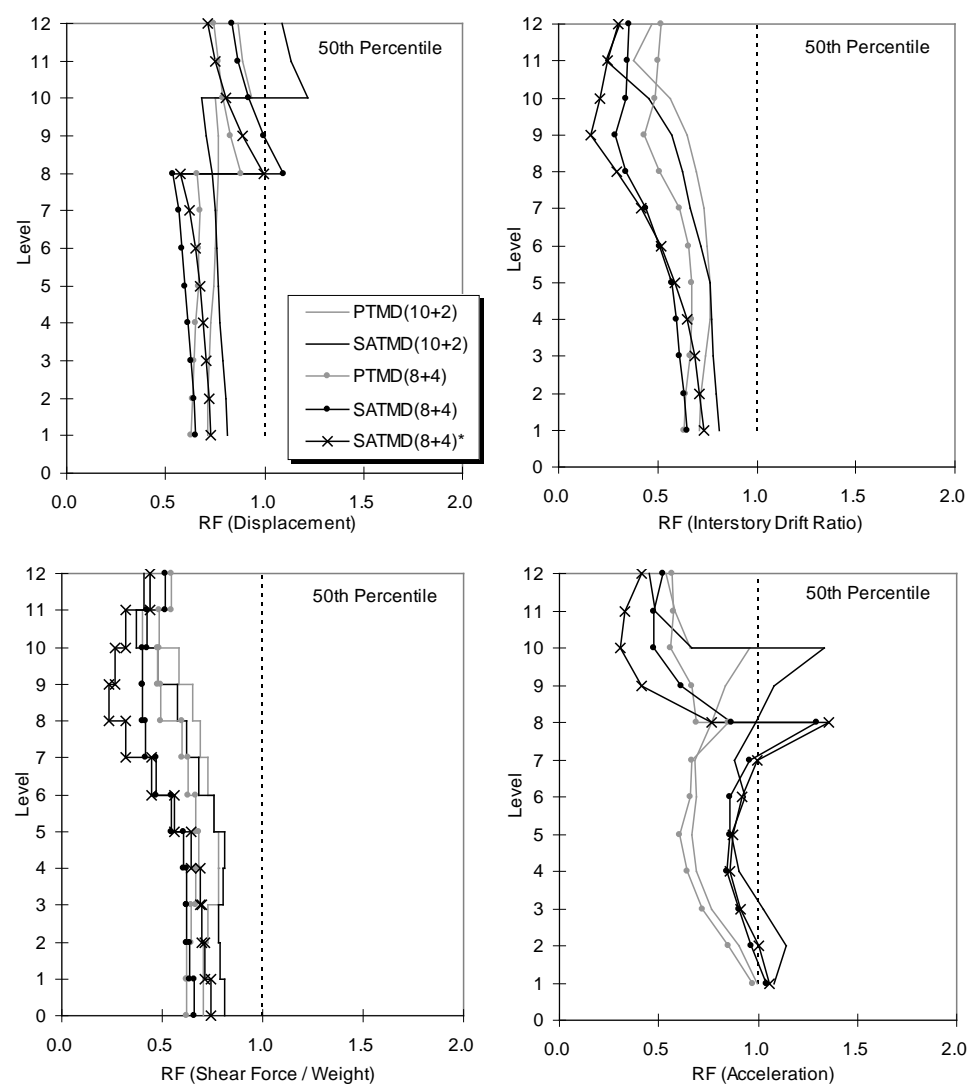

Figure 11. High suite response reduction factors of ' $10+2$ ' and ' $8+4$ ' models ( $50^{\text {th }}$ percentile)

\subsection{Seismic performance results}

Modal analysis showed that the TMD building systems have the unique modal features to isolate the structure to be controlled effectively and that the resetable device provides a more advanced control function by effectively anticipating the isolation layer response using sensor feedback. Specifically, the PTMD and SATMD response clearly showed a far different dominant structural period of response compared to the uncontrolled case. Hence, the modal response between the TMD systems used shows that the PTMD and SATMD designs developed reduce structural response by different mechanisms, which is an interesting and unique result of its own.

From the performance results based on the several response indices, the time history analysis and normalized reduction factor results showed that TMD building systems present significant reductions on the control indices to all seismic hazards. In this respect, the SATMD and the higher mass ratio $(8+4)$ building structures, as compared to the PTMD building system, have shown the attractive results. However, the acceleration response of the PTMD is better than that of the SATMD due to the PTMD damping provided. Despite of this particular point, almost all results show the ability of the SA device and larger mass ratio $(8+4)$ to reduce overall structural response measures. In particular, the reduction of seismic demands for these cases is most pronounced in the $84^{\text {th }}$ percentile responses.

The maximum displacements of each level increase steadily over the height of the level and the control effects of the displacement are proportional as the height of the building. Large displacements can be found at the isolation layer, especially in the 
SATMD system and this tendency has been expected from the previous modal properties of the almost separated modal responses and the increased participation factor of the second mode [9]. They also maximize the effect of the SA devices to the best effect within this design.

The better control effects of the SATMD and the higher mass ratio $(8+4)$ building structures compared to the PTMD building system can be seen in the response of interstory drift and shear force at mid and higher floor levels. This tendency is increased for the larger intensity, primarily near-field, high suite ground motions. For the interstory drift, the low suite induces median interstory drift demands, as a representative value, of about $0.5 \%$. This value increases to about $1 \%$ and $2 \%$ under the medium and high suites respectively. For the No TMD (conventional ductile) structure, the location of peak interstory drift occurs in the $9^{\text {th }}$ floor. For the TMD building structures, however, the interstory drifts are distributed constantly or proportionally over the floor level under the suites. From the statistical response of the interstory drifts and story shear forces, it is apparent that the upper stories above the isolation interface of the SATMD building system are effectively controlled due to the proper interrupting functions of the SA isolation system from the seismic energy. As the earthquake intensity increases the effectiveness of the drift reduction of SATMD becomes more effective, particularly compared to the PTMD. This is because the SATMD is better able to cope with the detuning effects of nonlinear response.

The acceleration responses of the isolated stories of the upper segment have a significant reduction in all cases. The reason for these reductions is that the upper segment is isolated from the main structure, so the base excitation is not transferred to the isolated upper portion directly. However, the acceleration response at the isolation interface of the SATMD system is clearly increased, but these are not felt by the occupants as the floor accelerations are modest at all times. What may be a problem is any equipment/piping etc. hung from the ceiling just below the segmented floor where the high accelerations occur. To solve the problems at the interface between the upper and lower segments, swivel joint or flexible metal/rubber joint, external wall with horizontal slit and deflection-smoothening mechanism have been suggested and installed by Kawamura et al. [36]. Furthermore, Charng [37] suggested three possible means of the linking design at the interface to prevent the occurrence of rocking modes of the structure and to transmit the gravity loads between the two segments.

A final point to note is that the SATMD systems appear more robust in Figures 6-8 in particular. The $50^{\text {th }}$ and $84^{\text {th }}$ percentile responses shown have similar displacements. In contrast, as expected the PTMD has generally higher values for the higher percentile response. This result indicates that the SATMD system, given its active component, has a more robust or adaptive affect and thus is more consistent across the suites of ground motions. Note that results are more similar in the high suite case where the resetable devices are saturating and thus providing a more limited impact for these very large events (see Figure 4). Hence, the overall bandwidth of control (difference between percentiles) is smaller, indicating more consistent performance for the SATMD systems, as detailed in further analyses in [38, 39].

Overall, the reduction factor profiles show that the TMD systems are more effective under higher intensity of the earthquake used. For the displacement reduction factors, as seen in the previous performance results, the values of relatively large response behavior are seen in the stories above the isolation layer. However, since these large reduction factors of over 1.0 are affected by large displacement at the isolation layer, the 
displacement within each segment of upper and lower story is relatively small. For the interstory drifts and shear force, the reduction factors of the isolated upper stories clearly indicate the advantage of the structural operation of the SATMD building systems, and the profiles indicate that the closer to the level of the isolation layer, the more less the reduction factor, especially for the medium and high suites. Similar as the previous performance results, the acceleration reduction factor profiles present the better seismic control performances for the upper isolated stories by the SATMD system and for the lower stories by the PTMD system respectively.

A last issue of concern is the displacement across the isolation layer seen particulary in Figures 6-8. This is an important consideration, given non-linear P-Delta affects that might arise, as well as the need to control the total motion of the upper segmented stories. Figures 6-8 show that the SATMD displaces more than the PTMD, but less than the No TMD case. The SATMD* displaces much further due to its much lower stiffness allowing greater displacement over the isolation layer. However, it should be noted that the resetable devices rely on large displacement to mitigate energy. Thus, the SATMD system is behaving as desired. In contrast, the PTMD systems rely on velocity across the interface and their viscous dampers to mitigate energy. Thus, the systems remove energy far differently.

Given the median SATMD cases presented being similar or smaller than the No TMD cases for the Low and Medium suites, it is not unfair to conclude that such drift over the isolation layer is not necessarily a significant issue. In particular, the very large 65-85\% damping ratio provided for the optimum PTMD case may not be feasible in practical cases, resulting in larger PTMD responses and providing a further advantage for the SATMD concept. In fact, a similar result was obtained in a brief analysis of this issue in Mulligan et al [38]. More importantly, [38] showed that simple SATMD control laws, different than the 1-4 law used here and shown in Figure 4, could provide similar quality of control but dramatically reduce the isolation drift to similar levels as the No TMD or PTMD case, by changing the resetable device feedback control laws to focus on the isolation layer drift as a control metric, rather than the motion of the top base structural story. Hence, the issue, while important, can be managed readily. Further these two case studies have highlighted this issue where the companion spectral analysis was not able to provide the necessary response detail.

Finally, it should be noted that both PTMD and SATMD systems have the same total isolating stiffness, $k_{M 2 o p t}$, and the PTMD results are optimal, but not necessarily practical. Specifically, the 65-85\% damping ratio for the PTMD system might not be really achieved. In addition, perfect PTMD tuning is rare and will affect performance. Thus, similar SATMD results indicate that optimal level solutions can be obtained without resorting to infeasibly large non-linear viscous dampers. Other studies with different stiffness values optimized to each case have shown clearer differences, especially if the PTMD is not ideally or perfectly tuned [38].

As discussed above, the modes of removing energy are very different for these two approaches to TMD systems. In particular, Table 4 shows that the SATMD systems have longer periods of response for the first mode matched with far different mass and modal participation factors for the first and second modes. More specifically, the second mode participates more in the response of the SATMD than for the PTMD case (60$75 \% 1^{\text {st }}$ mode response for the PTMD relative to the second mode, and 50\% for the first mode in the SATMD cases in Table 4). Additionally, as noted, one system maximizes dissipation via maximizing dissipation across the resetable devices (SATMD), while the 
PTMD is most effective when velocities across the dissipation layer are a maximum. Hence, the SATMD can be seen to mitigate energy by shifting response across modes and lowering effective structural periods in the response [39], in contrast to the PTMD which still has a first mode dominant response, as expected. The exact reasons, beyond the modeling used are not fully known and will require greater investigation, but dynamically, it is clear that the two systems remove energy differently both at a device level and, via less obvious mechanisms, over the whole structure.

The overall hypothesis of this research has been that an SATMD system with a similar overall stiffness as a PTMD system can be parametrically optimized to provide good performance that is potentially more robust to tuning errors. Thus, to reduce optimisation variables and optimisation non-convexity and non-linearity, we have initially hypothesized that the optimal PTMD stiffness represents a good or acceptable design baseline. This choice is much like choosing a starting value for any non-linear optimization problem. In this case, we have chosen start value and a parametric optimisation approach to SATMD stiffness contributions to define the variable space and yield a highly computationally efficient and convex approach to designing such systems. Therefore, even though this study does not provide exact design criteria, the aim of this analysis is to statistically quantify the fundamental qualitative benefit of these TMD systems by examining both the efficacy of the segregated structural configuration and the use of resetable devices in that approach. The response features obtained in this linear analysis can be used as the initial design reference for the further studies investigating inelastic seismic response for more realistic nonlinear structures.

Finally, this paper has presented two realistic retrofit or height extension applications that might arise in the design of structures for growing modern cities, as well as for novel new structures. The value of these application examples is primarily in their difference from the companion presentation of spectral analyses. This paper has provided significant insight into the complexities of such systems and their application. In particular, it has shown that at a specific case the response of a given system (e.g. SATMD or PTMD or SATMD*) may be better than the others at the median 50\% level for a given ground motion suite, but less effective at the $84 \%$ level, as shown in Figures 6-8 and 9-11. This variation highlights the need to do case specific dynamic analyses beyond what is available via simplified spectral analyses. Where spectral analyses have their place in providing overall design guidance and proof of concept, these specific analyses, as presented here, provide greater insight into specific details, their level of import versus traditional fixed design, and the potential for variability across suites of ground motions at a given likelihood of occurrence.

\section{CONCLUSIONS}

This research presented a case study on the seismic response of linear multi-story passive and semi-active tuned mass damper building systems under probabilistically scaled suites of earthquake records. Hence, they provide good initial indications of the efficacy of any design approach. To demonstrate the effects of the PTMD and SATMD building systems, $10+2$ and $8+4$ story, two-bay reinforced concrete framed structures were developed. The optimal parameters of the MDOF structure were demonstrated and a reasonable stiffness allocation to the resetable device and rubber bearings was processed.

The details and results of a set of comparative studies are used to assess the 
feasibility and effectiveness of such isolation systems. From the results of this comparative study, it is found that the proposed scheme may significantly reduce the seismic response of a structure. For example, the parameters of the interstory drift reduction factors are best reduced by SATMD building system. The acceleration responses of the isolated upper stories have a significant reduction in all cases. The reason for these reductions is that the upper stories are almost isolated from the main structure. However, the acceleration response of main stories is increased due to the operation of resetable device and this point needs to be considered in this type of TMD design. In view of these findings, and the fact that they might be relatively easy to construct using these emerging SA devices, it is concluded that the proposed SATMD building system has the potential to become a practical and effective way to reduce earthquake damage. Thus, these systems merit further studies to examine their advantages and to further develop experimental validation and design solutions, leading eventually to practical initial designs.

The development of designs suitable for implementing SATMD energy management systems ensure the proposed research remains focused on outcomes that are immediately useful. All such outcomes will advance the state of the art by providing additional knowledge and capability from which structural designers can draw in developing new structures or retrofitting existing structures. Finally, these outcomes ensure that the overall goal of taking semi-active energy management systems from a status of zero, or occasional highly specialized implementations, to a state where regular implementation may be more immediately practicable.

A trend towards widespread application of seismic isolation in civil engineering is underway. The concept of TMD systems can be equally well utilized for both segmented structure and traditional additional mass systems. As a method of structural retrofit, the additional stories would be added to an existing structure as these stories become a tuned mass, thus alleviating the necessity for additional mass. Furthermore, the segregated TMD building concept appears to hold the promise of modifying the structural configuration of irregular structures that result from non-uniform mass, stiffness, strength, structural form, or a combination. It also extends the technique of base isolation to taller buildings and other types of seismic isolation-based control strategy. Structural control provides an extra mechanism to improve seismic structural performance. For maximum effectiveness, minimal control effort is required to achieve the desired performance goals. Based on this point of view, this research has demonstrated the validity of the realistic PTMD and SATMD building systems for consideration in future design and construction. 


\section{REFERENCES}

1. Wang AP, Fung RF, Huang SC. Dynamic analysis of a tall building with a tuned-mass-damper device subjected to earthquake excitations. Journal of Sound and Vibration 2001; 244(1):123-136.

2. Sadek F, Mohraz B, Taylor AW, Chung RM. A method of estimating the parameters of tuned mass dampers for seismic applications. Earthquake Engineering \& Structural Dynamics 1997; 26(6):617635.

3. Li Q, Cao H, Li G, Li S, Liu D. Optimal design of wind-induced vibration control of tall buildings and high-rise structures. Wind and Structures, An International Journal 1999; 2(1):69-83.

4. Aldemir U. Optimal control of structures with semiactive-tuned mass dampers. Journal of Sound and Vibration 2003; 266(4):847-874.

5. Chang CC, Yang HTY. Control of buildings using active tuned mass dampers. Journal of Engineering Mechanics 1995; 121(3):355-366.

6. Li C, Liu Y, Wang Z. Active multiple tuned mass dampers: A new control strategy. Journal of Structural Engineering 2003; 129(7):972-977.

7. Ricciardelli F, Occhiuzzi A, Clemente P. Semi-active Tuned Mass Damper control strategy for wind-excited structures. Journal of Wind Engineering and Industrial Aerodynamics 2000; 88(1):5774.

8. Mar D, Tipping S. Story Isolation: A New High-Performance Seismic Technology. 9th US-Japan Workshop on the Improvement of Structural Design and Construction Practices, Victoria, British Columbia, Canada; 2000.

9. Ziyaeifar M, Noguchi H. Partial mass isolation in tall buildings. Earthquake Engineering \& Structural Dynamics 1998; 27(1):49-65.

10. Ziyaeifar M. Mass isolation, concept and techniques. European earthquake engineering 2002(2):4355.

11. Sueoka T, Torii S, Tsuneki Y. The Application of Response Control Design Using Middle-Story Isolation System to High-Rise Building. 13th World Conference on Earthquake Engineering, Vancouver, Canada; 2004, Paper No. 3457.

12. Feng MQ, Mita A. Vibration control of tall buildings using mega subconfiguration. Journal of Engineering Mechanics 1995; 121(10):1082-1088.

13. Zhou FL, Yang Z, Liu WG, Tan P. New seismic isolation system for irregular structure with the largest isolaton building area in the world. 13th World Conference on Earthquake Engineering, Vancouver, Canada; 2004.

14. Zhu H, Wen Y, Iemura H. A study on interaction control for seismic response of parallel structures. Computers \& Structures 2001; 79(2):231-242.

15. Pan T-C, Ling S-F, Cui W. Seismic response of segmental buildings. Earthquake Engineering \& Structural Dynamics 1995; 24(7):1039-1048.

16. Murakami K, Kitamura, H., Ozaki, H., and Teramoto, T. Design and analysis of a building with the middle-story isolation structural system. 12th World Conference of Earthquake Engineering. Auckland, New Zealand; 2000, Paper No. 0857.

17. Chey MH, Carr AJ, Chase JG, Mander JB. Design of Semi-Active Tuned Mass Damper Building Systems using Resetable Devices. 8th Pacific Conference on Earthquake Engineering, Singapore; 2007.

18. Carr AJ. RUAUMOKO. Computer Program Library: Department of Civil Engineering, University of Canterbury; 2004.

19. Jury RD. Seismic load demands on columns of reinforced concrete multistorey frames. Masters Thesis. Christchurch: Department of Civil Engineering, University of Canterbury; 1978.

20. NZS4203. New Zealand Standard; Code of Practice for General Structural Design and Design Loadings for Buildings. Standards Association of New Zealand (SANZ), 1976.

21. NZS4203. New Zealand Standard; Code of Practice for General Structural Design and Design Loadings for Buildings, Vol.1 Code of Practive and Vol.2 Commenraty Standards Association of New Zealand (SANZ), 1992.

22. Paulay T. Moment redistribution in continuous beams of eqrthquake resistant multistorey reinforced concrete frames. Bulletin of the New Zealand National Society of Earthquake Engineering 1976; 9(4):205-212. 
23. Thomson ED. P-delta effects in ductile reinforced concrete frames under seismic loading. Masters Thesis, Christchurch: Department of Civil Engineering, University of Canterbury; 1991.

24. Paulay T. Developments in the Design of Ductile Reinforced Concrete Frames. Proc of the 2nd South Pacific Regional Conference on Earthquake Engineering, Wellington, New Zealand; 1979. p. 605-623.

25. Mulligan K, Chase, JG, Mander, JB, Fougere, M, Deam, BL, Danton, G and Elliott, RB. Hybrid experimental analysis of semi-active rocking wall systems. Proc New Zealand Society of Earthquake Engineering 2006 Conference (NZSEE), Napier, New Zealand; 2006.

26. Hunt SJ. Semi-active smart-dampers and resetable actuators for multi-level seismic hazard mitigation of steel moment resisting frames. Masters Thesis, Christchurch: Department of Mechanical Engineering, University of Canterbury; 2002.

27. Chase JG, Mulligan KJ, Gue A, Alnot T, Rodgers G, Mander JB, Elliott R, Deam B, Cleeve L, Heaton D. Re-shaping hysteretic behaviour using semi-active resetable device dampers. Engineering Structures 2006; 28(10):1418-1429.

28. Rodgers GW, Mander JB, Chase JG, Mulligan KJ, Deam BL, Carr A. Re-shaping hysteretic behaviour - Spectral analysis and design equations for semi-active structures. Earthquake Engineering \& Structural Dynamics 2007; 36(1):77-100.

29. Bobrow JE, Jabbari F, Thai K. A new approach to shock isolation and vibration suppression using a resetable actuator. Journal of Dynamic Systems, Measurement and Control, Transactions of the ASME 2000; 122(3):570-573.

30. Barroso LR, Chase JG, Hunt S. Resetable smart dampers for multi-level seismic hazard mitigation of steel moment frames. Journal of Structural Control 2003; 10(1):41-58.

31. Jabbari F, Bobrow JE. Vibration suppression with resetable device. Journal of Engineering Mechanics 2002; 128(9):916-924.

32. Kurino H, Yamada T, Matsunaga Y, Tagami J. Switching Oil Damper with Automatic Valve Operation System for Structural Control. 4th World Conference on Structural Control and Monitoring, University of California, San Diego; 2006.

33. Shmizu K, Orui S, Kurino H. Observed Response of the High-Rise Building with Semi-Active Switching Oil Dampers to Earthquake and Typhoon. 4th World Conference on Structural Control and Monitoring, University of California, San Diego; 2006.

34. Sommerville P, Smith, N., Punyamurthula, S., and Sun, J. Development of ground motion time histories for Phase II of the FEMA/SAC steel project. SAC Back-ground Document Report No SAC/BD-97/04; 1997.

35. Limpert E, Stahel WA, Abbt M. Log-normal distributions across the sciences: Keys and clues. Bioscience 2001; 51(5):341-352.

36. Kawamura S, Sugisaki R, Ogura K, Maezawa S, Tanaka S, Yajima A. Seismic isolation retrofit in Japan. 12th World Conference on Earthquake Engineering, Auckland, New Zealand; 2000, Paper No. 2523.

37. Charng P-H. Base isolation for multistorey building structures. Ph.D. Thesis, Christchurch: Department of Civil Engineering, University of Canterbury; 1998.

38. Mulligan K, Miguelgorry, M, Novello, V, Chase, JG, Mander, JB, Rodgers, GW, Carr, AJ, Deam, BL and Horn, B. Semi-active Tuned Mass Damper Systems. 19th Australasian Conference on Mechanics of Structures and Materials (ACMSM), Christchurch, New Zealand; 2006.

39. Chey, MH (2007) " Passive And Semi-Active Tuned Mass Damper Building Systems," PhD Thesis, University of Canterbury, Dept of Civil Engineering, Christchurch, New Zealand. 
Table 1

Dynamic properties of 8 storey, 10 storey and 12-storey buildings

\begin{tabular}{lcccl}
\hline Item & 8-storey & 10-storey & 12-storey & Unit \\
\hline Weight & 12,940 & 16,080 & 19,190 & $\mathrm{kN}$ \\
$1^{\text {st }}$ Modal Mass & 1,072 & 1,301 & 1,514 & tone (Mg) \\
Natural period & 1.187 & 1.518 & 1.880 & $\mathrm{sec}$ \\
Frequency & 5.30 & 4.14 & 3.34 & $\mathrm{rad} / \mathrm{sec}$ \\
Damping Ratio & 0.05 & 0.05 & 0.05 & - \\
$1^{\text {st }}$ Modal Amplitude & 1.309 & 1.343 & 1.366 & - \\
\hline
\end{tabular}


Table 2

Member sizes of the framed structure

\begin{tabular}{lcc}
\hline Members & Level & Dimensions $(\mathrm{mm})$ \\
\hline \multirow{3}{*}{ Beams } & $1-6$ & $900 \times 400$ \\
& $7-8$ & $850 \times 400$ \\
& $9-12$ & $800 \times 400$ \\
\hline \multirow{2}{*}{ Exterior } & $1-6$ & $775 \times 500$ \\
Columns & $7-8$ & $750 \times 500$ \\
& $9-12$ & $650 \times 500$ \\
\hline \multirow{2}{*}{ Interior } & $1-6$ & $800 \times 800$ \\
Column & $7-8$ & $725 \times 725$ \\
& $9-12$ & $675 \times 675$ \\
\hline
\end{tabular}


Table 3

Parameters for TMD building systems

\begin{tabular}{lcccccc}
\hline Model & $\mu$ & $f_{M 2 o p t}$ & $\xi_{M 2 o p t}$ & $\begin{array}{c}k_{M 2 o p t} \\
(\mathrm{kN} / \mathrm{m})\end{array}$ & $\begin{array}{c}c_{M 2 o p t} \\
(\mathrm{kN}-\mathrm{s} / \mathrm{m})\end{array}$ & $\begin{array}{c}\text { Device force } \\
(\mathrm{kN})\end{array}$ \\
\hline PTMD(10+2) & 0.244 & 0.734 & 0.649 & 2,935 & 1,252 & - \\
SATMD(10+2) & 0.244 & 0.734 & - & 2,935 & - & 644 \\
PTMD(8+4) & 0.594 & 0.544 & 0.840 & 5,293 & 3,085 & - \\
SATMD(8+4) & 0.594 & 0.544 & - & 5,293 & - & 1,573 \\
\hline
\end{tabular}


Table 4

Numerical results of modal analysis

\begin{tabular}{|c|c|c|c|c|c|}
\hline \multirow{2}{*}{ TMD } & \multirow{2}{*}{ Mode } & \multirow{2}{*}{$\begin{array}{l}\text { Mass } \\
\text { (tone) }\end{array}$} & \multirow{2}{*}{$\begin{array}{r}\text { Frequency } \\
\text { (rad/sec) }\end{array}$} & \multicolumn{2}{|c|}{ Part-Fact } \\
\hline & & & & mode & mass \\
\hline \multirow{3}{*}{ No TMD } & $1^{\mathrm{st}}$ & 1514 & 0.53 & 1.37 & 0.805 \\
\hline & $2^{\text {nd }}$ & 252 & 1.52 & -0.53 & 0.134 \\
\hline & $3^{\text {rd }}$ & 74 & 2.73 & -0.27 & 0.039 \\
\hline \multirow{3}{*}{$\begin{array}{l}\text { PTMD } \\
(10+2)\end{array}$} & $1^{\mathrm{st}}$ & 816 & 0.38 & 1.53 & 0.436 \\
\hline & $2^{\text {nd }}$ & 812 & 0.74 & 0.94 & 0.434 \\
\hline & $3^{\text {rd }}$ & 181 & 1.92 & -0.50 & 0.097 \\
\hline \multirow{3}{*}{$\begin{array}{l}\text { SATMD } \\
(10+2)\end{array}$} & $1^{\text {st }}$ & 513 & 0.27 & 1.27 & 0.274 \\
\hline & $2^{\text {nd }}$ & 1109 & 0.68 & 1.20 & 0.593 \\
\hline & $3^{\text {rd }}$ & 187 & 1.90 & -0.50 & 0.100 \\
\hline \multirow{3}{*}{$\begin{array}{l}\text { PTMD } \\
(8+4)\end{array}$} & $1^{\text {st }}$ & 1020 & 0.36 & 1.29 & 0.541 \\
\hline & $2^{\text {nd }}$ & 697 & 0.96 & 0.97 & 0.370 \\
\hline & $3^{\text {rd }}$ & 39 & 2.39 & 0.28 & 0.021 \\
\hline \multirow{3}{*}{$\begin{array}{l}\text { SATMD } \\
(8+4)\end{array}$} & $1^{\mathrm{st}}$ & 834 & 0.27 & 1.17 & 0.442 \\
\hline & $2^{\text {nd }}$ & 878 & 0.89 & 1.15 & 0.465 \\
\hline & $3^{\text {rd }}$ & 47 & 2.33 & -0.30 & 0.025 \\
\hline
\end{tabular}

